\title{
Non-Exposed Endoscopic Wall-Inversion Surgery for Gastrointestinal Stromal Tumor of the Stomach: First Case Report in Korea
}

\author{
Da Won Kim¹, Joon Sung Kim¹, Byung-Wook Kim¹, Ji Yun Jung' ${ }^{1}$ Gi Jun Kim¹ and Jin-Jo Kim² \\ ${ }^{1}$ Division of Gastroenterology, Department of Internal Medicine, ${ }^{2}$ Department of General Surgery, Incheon St. Mary's Hospital, College of Medicine, \\ The Catholic University of Korea, Incheon, Korea
}

Laparoscopic wedge resection of the stomach is a widely accepted treatment for primary resectable gastrointestinal stromal tumors (GISTs). However, it is difficult to determine the appropriate incision line from outside of the stomach, and many attempts have been made to avoid unnecessary resection of unaffected gastric tissues. Recently a technique called non-exposed endoscopic wall-inversion surgery (NEWS) was introduced to avoid exposure of GIST to the peritoneum. Here, we describe the first published case of NEWS for GIST of the stomach practiced in Korea. Clin Endosc 2016;49:475-478

Key Words: Gastrointestinal stromal tumors; Stomach; Endoscopy; Laparoscopy

\section{INTRODUCTION}

Laparoscopic wedge resection of the stomach is a widely accepted treatment for primary resectable gastrointestinal stromal tumors (GISTs) due to the technical efficacy of linear staplers. ${ }^{1}$ However, it is difficult to determine the appropriate incision line from outside of the stomach when the lesions are intraluminal. This may result in unnecessary or excessive excisions. ${ }^{2}$ Many attempts have been made to avoid unnecessary resection of unaffected gastric tissues, including endoscopic full-thickness resection (EFTR), classic laparoscopic and endoscopic cooperative surgery (LECS), and modified LECS procedures. ${ }^{2}$ Recently, a technique called non-exposed endoscopic wall-inversion surgery (NEWS) was introduced to avoid exposure of GIST to the peritoneum. ${ }^{3}$ In the present case, we describe, to our knowledge, the first case of NEWS

Received: November 30, 2015 Revised: February 1, 2016

Accepted: February 16, 2016

Correspondence: Byung-Wook Kim

Division of Gastroenterology, Department of Internal Medicine, Incheon St. Mary's Hospital, College of Medicine, The Catholic University of Korea, 56 Dongsu-ro, Bupyeong-gu, Incheon 21431, Korea

Tel: +82-32-280-5052, Fax: +82-32-280-5987, E-mail: gastro@catholic.ac.kr

(cc) This is an Open Access article distributed under the terms of the Creative Commons Attribution Non-Commercial License (http://creativecommons.org/ licenses/by-nc/3.0) which permits unrestricted non-commercial use, distribution, and reproduction in any medium, provided the original work is properly cited. for GIST of the stomach practiced in Korea, with a review of the literature.

\section{CASE REPORT}

A 67-year-old woman visited our department for evaluation of a gastric mass. The mass was discovered during gastroscopy as part of a routine health checkup and was defined as a $2.0 \times 1.5$ $\mathrm{cm}$ subepithelial tumor at the greater curvature side of the fundus base (Fig. 1A). Endoscopic ultrasonography revealed that the lesion originated from the 4 th muscle layer (proper muscle layer) with a homogeneous and hypoechoic pattern (Fig. 1B).

A computed tomography scan showed that the lesion protruded into the gastric lumen, with no evidence of distant or lymph node metastasis (Fig. 2). The patient was informed of several surgical treatment options and agreed to undergo NEWS.

NEWS was performed as follows. Under general anesthesia, the patient was placed in the supine position. A $12-\mathrm{mm}$ camera port was inserted into the umbilicus. Subsequently, 5 $\mathrm{mm}$ trocars were placed in the right upper, left upper, and left lower quadrants, and a $12 \mathrm{~mm}$ trocar was placed in the right lower quadrant. Several serosal markings were made laparo- 

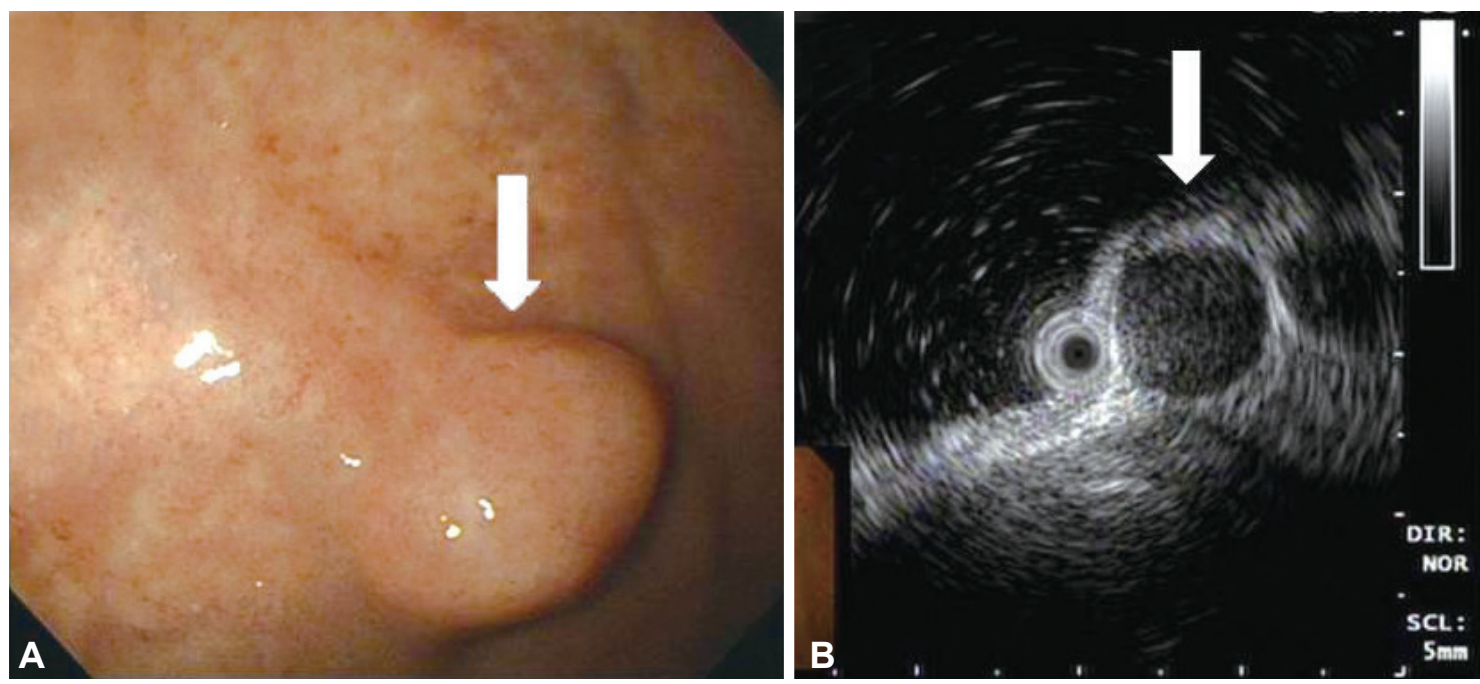

Fig. 1. (A) Initial gastroscopy. The image shows a subepithelial lesion, approximately $2.0 \times 1.5 \mathrm{~cm}$ in size, at the greater curvature side of the fundus base (arrow). (B) Endoscopic ultrasound shows that the lesion originated from the 4th muscle layer with a homogeneous and hypoechoic pattern (arrow).

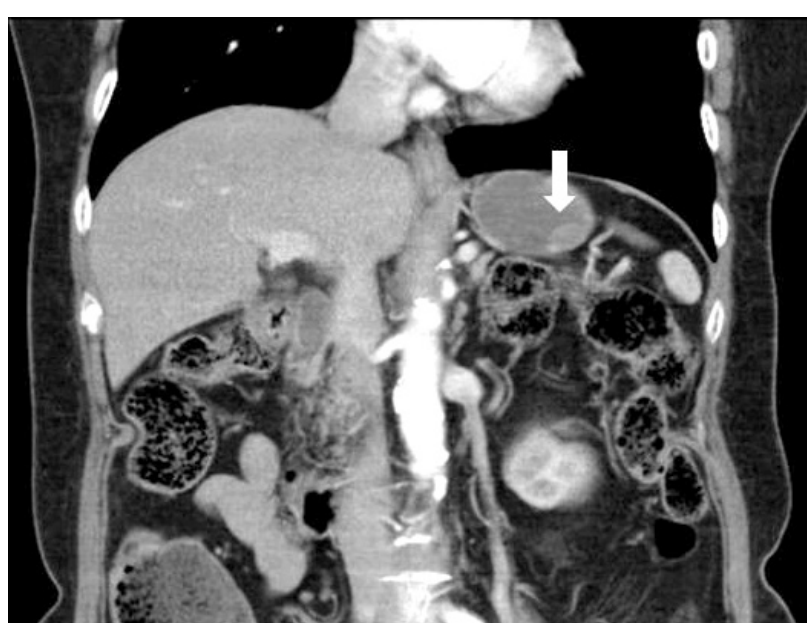

Fig. 2. Abdomen computed tomography. The image shows a subepithelial lesion protruding into the gastric lumen (arrow).

scopically, and a circumferential seromuscular incision was made with electrocautery. After creating a flap, the seromuscular layers were vertically sutured in an interrupted manner using vicryl thread to invert the lesion into the gastric lumen (Fig. 3). The lesion was removed by endoscopic submucosal dissection (ESD) using a dual knife (Olympus, Tokyo, Japan), and the mucosal side was closed with clips. The detached lesion was removed perorally (Fig. 4). Potential air leakages or mucosal defects were investigated using laparoscopy.

The elapsed time from the serosal markings to endoscopic removal of the lesion was 40 minutes (serosal markings, 2 minutes; seromuscular incision, 9 minutes; suture, 14 minutes; ESD with retrieval, 15 minutes). There were no complications, and the estimated blood loss was less than $50 \mathrm{~mL}$. The surgical margin was clear and the capsule was covered with the mu- cosa and serosa. The final pathologic diagnosis was GIST of a very low risk. Immunohistochemical stains were positive for CD34 and CD117 (Fig. 5).

Ceftriaxone was administered for 5 days and the patient's diet was resumed 3 days after the procedure. Her condition improved, and she was discharged 10 days after the procedure.

\section{DISCUSSION}

EFTR is a minimally invasive endoluminal surgery for gastrointestinal tumors. However, a major limitation of this technique is that gastric contents can often flow into the peritoneal cavity during the procedure, which may cause viable cancer cell seeding in the peritoneal cavity. The concept of seromuscular incision was introduced by Inoue et al., ${ }^{4}$ who invented the combination of laparoscopic and endoscopic approaches to neoplasia with a non-exposure technique (CLEAN-NET). This has expanded the role of laparoscopic-endoscopic rendezvous surgery.

NEWS is a new technique introduced by Goto et al. ${ }^{3}$ This technique enables full-thickness resection of the gastric wall without transmural communication and can avoid intra-abdominal infection or tumor seeding to the peritoneum. This technique has been successfully performed for subepithelial tumors such as GIST and Schwannoma, and in early gastric cancer.,

NEWS has limitations. To remove the lesion perorally, the resected specimen should be less than $3 \mathrm{~cm}$ in size; when the lesion is located in areas such as the esophagogastric junction or near the pylorus, resection of the lesion is technically more difficult. The biggest disadvantage of the NEWS technique 

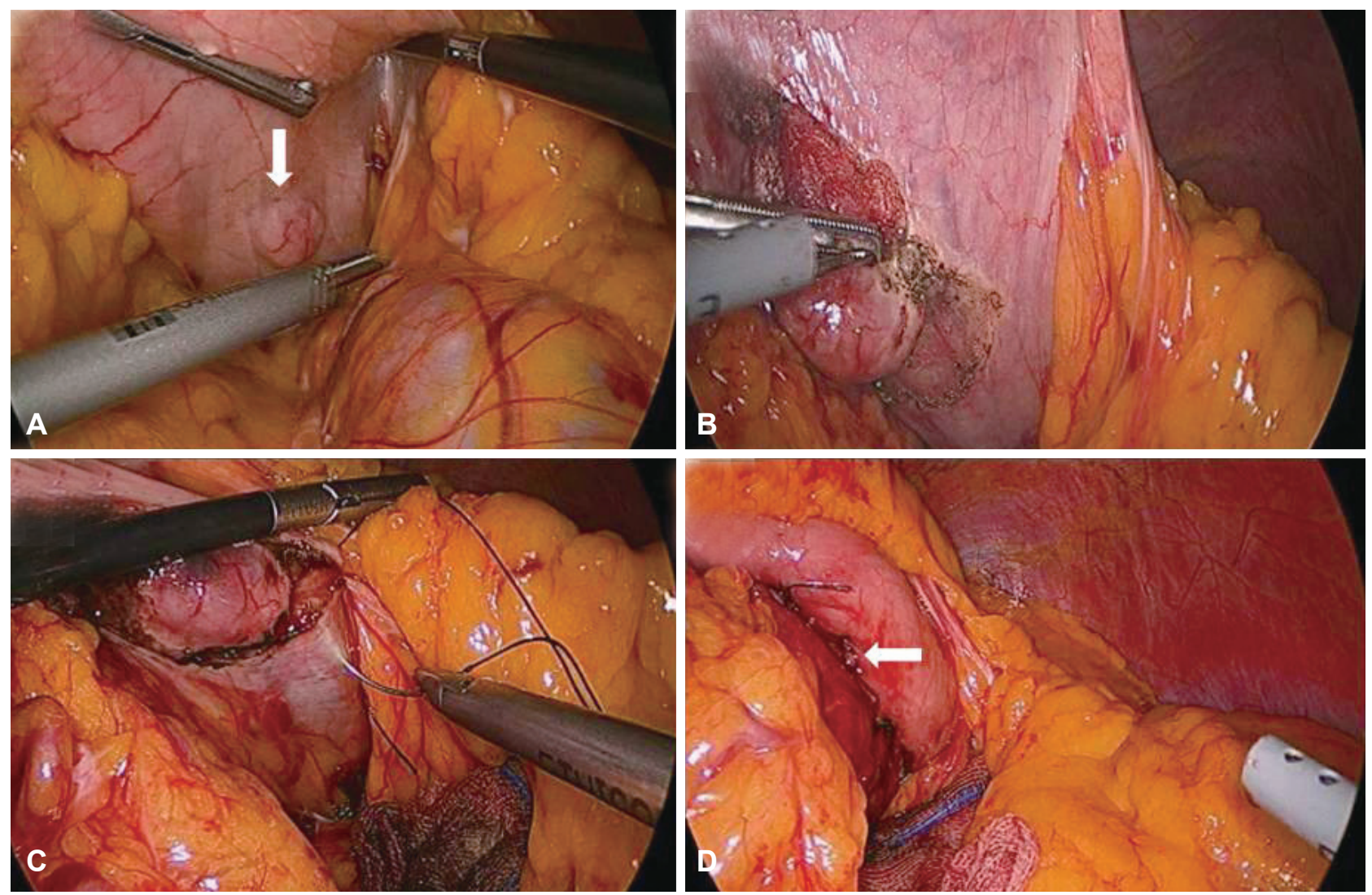

Fig. 3. Laparoscopic view. (A) The image shows the lesion protruding slightly into the peritoneal cavity (arrow). (B) Electrocautery of the circumferential seromuscular incision. (C) Suture of the seromuscular layers. (D) Complete suturing of the serosal surface (arrow).

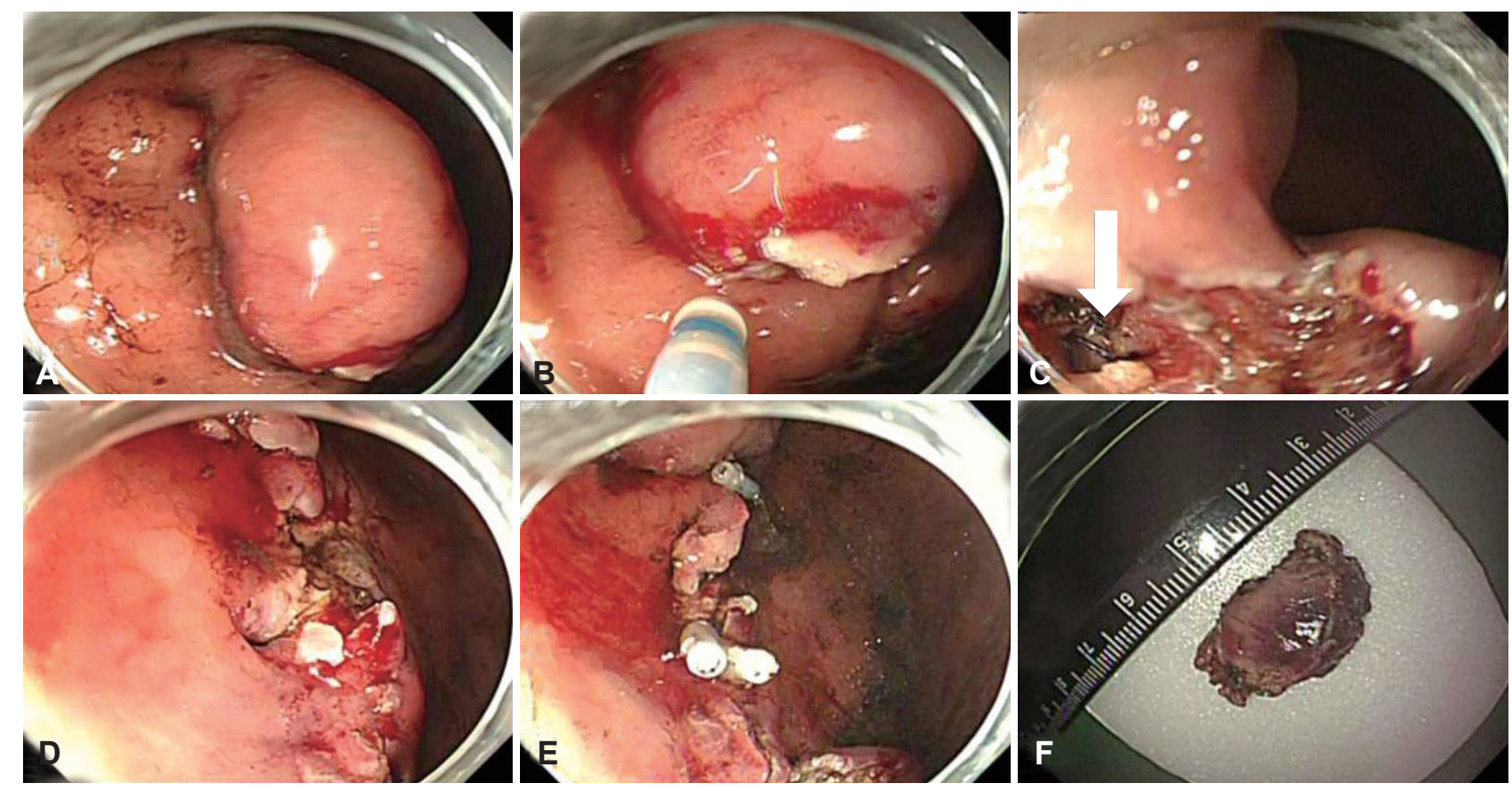

Fig. 4. (A) The inverted lesion protruding into the gastric lumen. (B) Submucosal dissection is performed with a dual knife. (C) Suture line during submucosal dissection (arrow). (D) Complete removal of the lesion. (E) The mucosal side is closed with clips. (F) The removed lesion measures $2.0 \times 1.5 \mathrm{~cm}$ in size. 

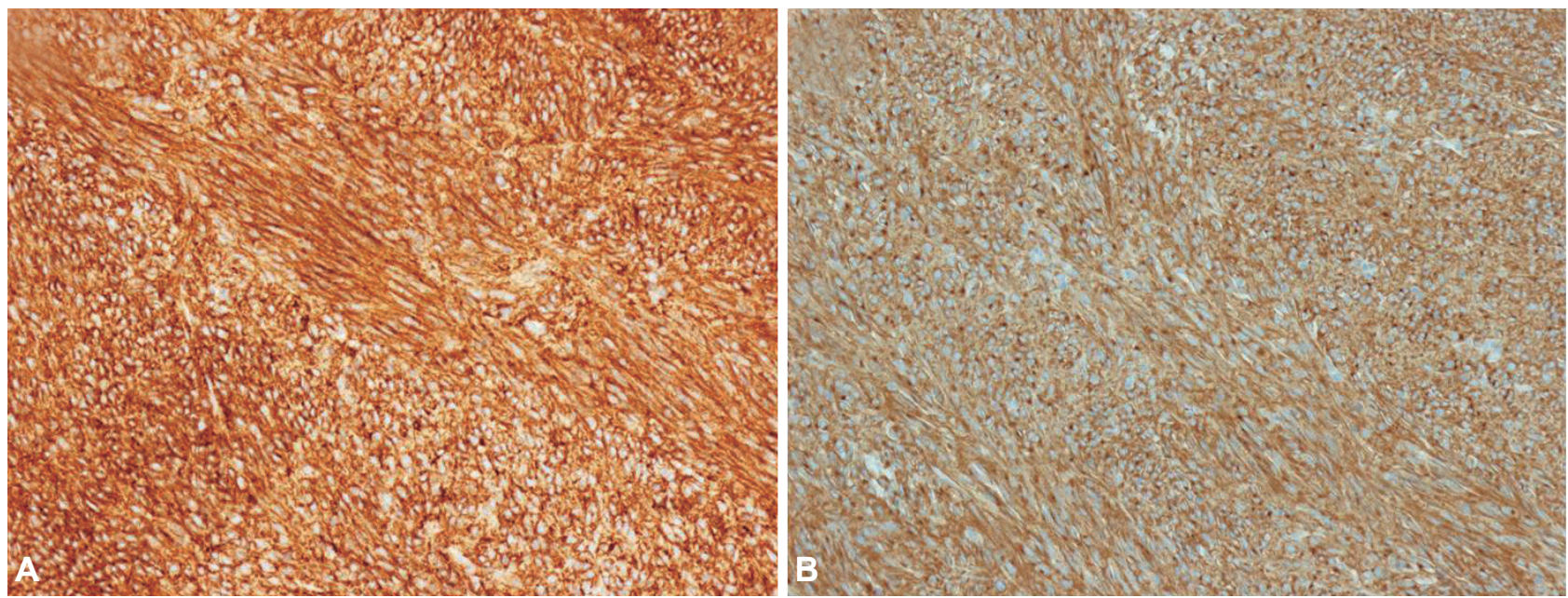

Fig. 5. Immunohistochemistry of the resected lesion. (A) Strong staining of CD34 is observed ( $\times 200)$. (B) Strong staining of CD117 (C-kit) is observed.

it is time-consuming. To overcome these limitations, several centers have been studying another type of EFTR.

Endoscopic tunnelling methods, such as submucosal tunnelling endoscopic resection (STER), represent other alternatives for the treatment of gastric GISTs. In STER, peritoneal punctures can be avoided, thereby saving time. However, a full-thickness lesion cannot be obtained, occasionally resulting in incomplete removal of the capsule. Furthermore, STER cannot be performed in some parts of the stomach.

To our knowledge, this is the first published case of NEWS in Korea. In the present case, the gastric GIST was successfully removed by NEWS without any complications. Histopathological examination showed that the whole GIST capsule was completely removed without rupture. Furthermore, the time taken for the entire procedure, including the laparoscopic and endoscopic procedures, was less than 40 minutes. During the dissection procedure, the suture line was identified laparoscopically, which suggests that a spacer (surgical sponge) is not always necessary in NEWS. By omitting this step, we were able to save time.

In summary, this is the first case of NEWS for GIST of the stomach performed in Korea. Further studies are required to confirm if NEWS can be established as a standard procedure for gastrointestinal tumors.
Conflicts of Interest

The authors have no financial conflicts of interest.

\section{REFERENCES}

1. Demetri GD, Benjamin RS, Blanke CD, et al. NCCN Task Force report: management of patients with gastrointestinal stromal tumor (GIST): update of the NCCN clinical practice guidelines. J Natl Compr Canc Netw 2007;5 Suppl 2:S1-S29.

2. Hiki N, Nunobe S, Matsuda T, Hirasawa T, Yamamoto Y, Yamaguchi T. Laparoscopic endoscopic cooperative surgery. Dig Endosc 2015;27:197204

3. Goto O, Mitsui T, Fujishiro M, et al. New method of endoscopic full-thickness resection: a pilot study of non-exposed endoscopic wall-inversion surgery in an ex vivo porcine model. Gastric Cancer 2011;14:183187.

4. Inoue H, Ikeda H, Hosoya T, et al. Endoscopic mucosal resection, endoscopic submucosal dissection, and beyond: full-layer resection for gastric cancer with nonexposure technique (CLEAN-NET). Surg Oncol Clin N Am 2012;21:129-140.

5. Mitsui T, Niimi K, Yamashita H, et al. Non-exposed endoscopic wall-inversion surgery as a novel partial gastrectomy technique. Gastric Cancer 2014;17:594-599.

6. Goto O, Takeuchi H, Kawakubo H, et al. First case of non-exposed endoscopic wall-inversion surgery with sentinel node basin dissection for early gastric cancer. Gastric Cancer 2015;18:434-439.

7. Ye LP, Zhang Y, Mao XL, et al. Submucosal tunnelling endoscopic resection for the treatment of esophageal submucosal tumours originating from the muscularis propria layer: an analysis of 15 cases. Dig Liver Dis 2013;45:119-123. 